\title{
Approximating fractional derivatives through the generalized mean
}

\author{
J.A. Tenreiro Machado, Alexandra M. Galhano, Anabela M. Oliveira, József K. Tar
}

a b s tract

This paper addresses the calculation of fractional order expressions through rational frac- tions. The article starts by analyzing the techniques adopted in the continuous to discrete time conversion. The problem is re-evaluated in an optimization perspective by tacking advantage of the degree of freedom provided by the generalized mean formula. The results demonstrate the superior performance of the new algorithm.

Keywords: Fractional derivatives, Fractional calculus, Average, Generalized mean

\section{Introduction}

Fractional calculus $(\mathrm{FC})$ deals with the generalization of integrals and derivatives to a non-integer order [1-5]. FC entails a wide field of applications by bringing into a broader paradigm concepts of physics, mathematics and engineering [6-10]. Nevertheless, FC is still an 'exotic' mathematical tool and its adoption in applied sciences requires some efforts towards the development of simple and clear algorithms.

One of the reasons for this state of affairs is the difficulty in applying FC, due to the higher complexity of the algorithms involved in the calculation of fractional derivatives. The generalization of the integrodifferential operator requires the adop- tion of approximations based on series or rational fraction expansions [9-17]. While the main volume of contributions has focused in getting expansion schemes, the problem of a systematic optimization procedure was not yet tackled.

In this line of thought, this paper addresses the optimal calculation of fractional order expressions tacking advantage of the extra parameters provided by the generalized averaging formula and is organized as follows. Section 2 introduces the calculation of fractional derivatives and formulates the problem in the perspective of generalized average formula. Section 3 presents a set of experiments that demonstrate the effectiveness of the proposed method. Finally, Section 4 outlines the main conclusions. 


\section{Problem formulation and adopted tools}

Since the foundation of the differential calculus the generalization of the concept of derivative and integral to a non-inte- ger order $a$ has been the subject of several approaches such as the Riemann-Liouville, the Grünwald-Letnikov, the Caputo and, based on transforms, the Fourier/Laplace definitions.

From the discrete-time point of view the Grünwald-Letnikov definition seems more attractive and, consequently, will be adopted in the sequel.

The Grünwald-Letnikov definition of a derivative of fractional order $a$ of the signal $x(t), D^{a} x(t)$, is

$$
D^{\alpha} x(t)=\lim _{h \rightarrow 0} \frac{1}{h^{\alpha}} \sum_{k=0}^{\infty} \frac{(-1)^{k} \Gamma(\alpha+1) x(t-k h)}{\Gamma(k+1) \Gamma(\alpha-k+1)}
$$

where $C$ is the gamma function and $h$ is the time increment. This formulation inspires a discrete-time calculation algorithm,based on the approximation of the time increment $h$ through the sampling period $T$, yielding the equation in the $z$ domain:

$$
Z\left\{D^{x} x(t)\right\} \approx\left[\frac{1}{T^{\alpha}} \sum_{k=0}^{\infty} \frac{(-1)^{k} \Gamma(\alpha+1)}{k \Gamma(\alpha-k+1)} z^{k}\right] X(z),
$$

where $X(z)=Z\{X(t)\}$.

The implementation of expression (2) corresponds to an $r$-term truncated series given by

$k$

$$
Z\left\{D^{z} x(t)\right\} \approx\left[\frac{1}{T^{2}} \sum_{k=0}^{r} \frac{(-1)^{k} \Gamma(\alpha+1)}{k \Gamma(\alpha-k+1)} z^{k}\right] X(z)
$$

In fact, expression (2) represents the Euler (or first backward difference) approximation in the so-called $s$ ? $z$ conversion scheme. Another possibility, often adopted in control system design, consists in the Tustin (or bilinear) rule. The Euler and Tustin rational

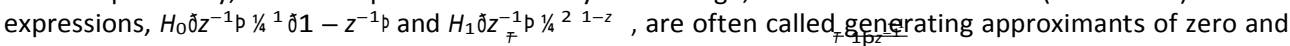

first order, respectively. Therefore, the generalization of these conversion methods leads to the non-integer order $a$ results:

$$
\begin{aligned}
& s^{\alpha} \approx\left[\frac{1}{T}\left(1-z^{-1}\right)\right]^{\alpha}=H_{0}^{z}\left(z^{-1}\right), \\
& s^{\alpha} \approx\left(\frac{2}{T} \frac{1-z^{-1}}{1+z^{-1}}\right)^{\alpha}=H_{1}^{z}\left(z^{-1}\right) .
\end{aligned}
$$

We can obtain a family of fractional differentiators generated by $H^{a} \delta z_{0}^{-1} p$ and $H^{a} \delta z^{-1} p$ weighted by the factors $p$ and $1-p$, yielding

$$
H_{a v}\left[z^{-1} ;(p, \alpha)\right]=p H_{0}^{z}\left(z^{-1}\right)+(1-p) H_{1}^{x}\left(z^{-1}\right)
$$

For example, the Al-Alaoui operator corresponds to an interpolation of the Euler and Tustin rules with weighting factor $p=3 / 4[18-20]$.

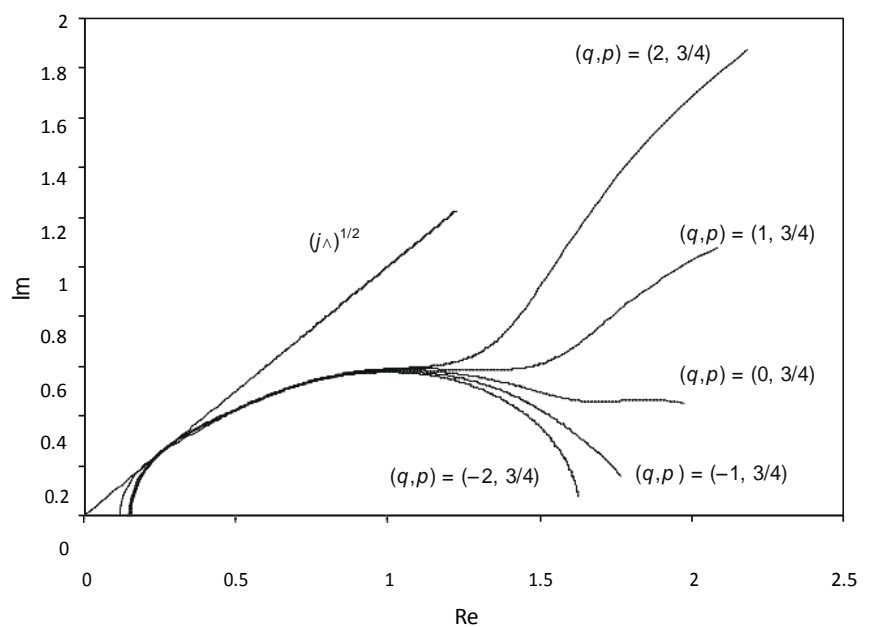

Fig. 1. Polar diagram of $D^{a}, a=1 / 2$ : the ideal case $(j X)^{a}$ versus the Padé fraction expansion $H_{k}(j X), k=4$, based on the averaging formulae $(6)$ with $q=\{-2,-1,0,1,2\}$ and $p=3 / 4, T=1,06 x<3 \mathrm{rad} \mathrm{s}^{-1}$. 
In order to get a rational expression, the final approximation corresponds to a truncated Taylor series or a rational fraction expansion. Due to its superior performance, often it is used a fraction of order $k 2 @$ :

$$
H_{k}\left(z^{-1}\right)=\frac{\sum_{i-1}^{k} a_{i} z^{-1}}{\sum_{i=0}^{k} b_{i} z^{-1}}, a_{i}, b_{i} \in \Re
$$

Moreover, usually it is adopted a Padé expansion in the neighborhood of $z=0$ and, since one parameter is linearly depen- dent, it is established $b_{0}=1$.

The arithmetic mean (4) motivates the study of an averaging method [21-24] based on the generalized formula of aver- ages (often called average of order $q 2 \mathrm{R}$ ):

$$
H_{a v}\left[z^{-1} ;(q, p, \alpha)\right]=\left\{p\left[H_{0}^{\alpha}\left(z^{-1}\right)\right]^{q}+(1-p)\left[H_{1}^{\alpha}\left(z^{-1}\right)\right]^{q}\right\}^{\frac{1}{4}},
$$

where $(q, p)$ are two tuning degrees of freedom, corresponding $q$ to the order of the averaging expression and $p$ to the weight-ing factor. Another possibility consists in the generalized $f$-mean that, applied to the generating functions, yields
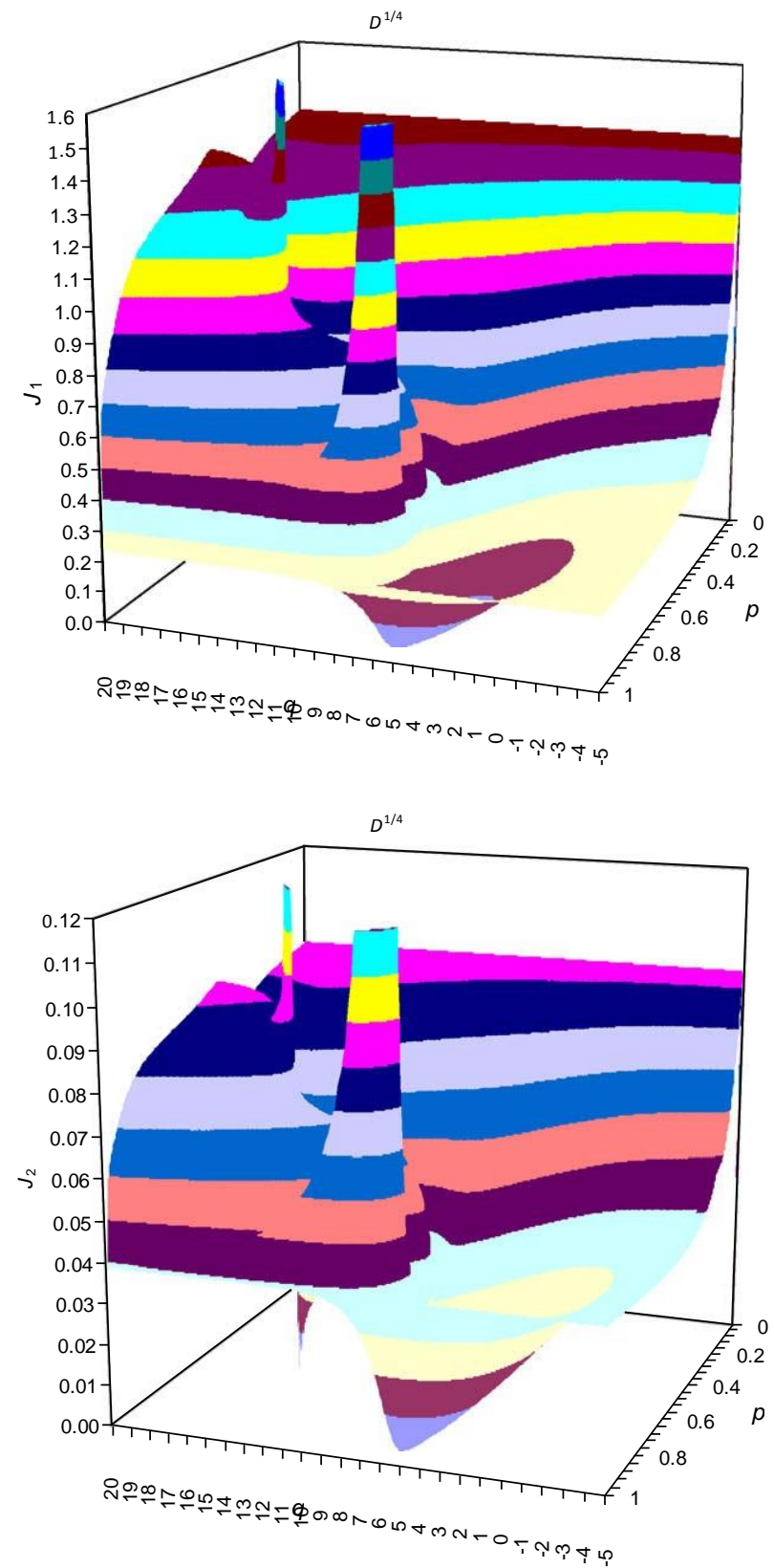

Fig. 2. Locus of $J_{i}(q, p), i=1,2$, for $D^{1 / 4}$ approximated through Padé fractions $H_{k}(j X), k=4$, based on the averaging formulae (6), with $T=1$, $06 X<3$ rad $\mathrm{s}^{-1}$ $N_{x}=300$. 
where $f$ is an invertible function that gives an extra design degree of freedom.

For example, when $q=\{-1,0,1\}$, in expression (6), or $f(x)=\left\{1 / x, e^{x}, x\right\}$ in expression (7), we get the well-known expressions for the \{harmonic, geometric, arithmetic\} averages.

\section{Approximate calculation of fractional derivatives}

In this section, we examine the expressions resulting from the generalized mean (6) when approximating $D^{a}, a=\{1 / 4,1 / 2,3 / 4\}$, through Padé fractions of order $k=4$. The comparison can be established either in the time or the frequency domains. Although conceptually equivalent, for the purpose of defining a optimization criteria in this paper it is adopted the frequency response and, therefore, is considered the transformation $z^{-1}=e^{-j X}, X=X T, j \frac{1}{4} 1$.

Fig. 1 depicts the polar diagram of $(j X)^{a}, a=1 / 2$, versus $H_{k}(j X), k=4$, based on the generalized mean (6) with $q=\{-2$, $-1,0,1,2\}$ and $p=3 / 4$, for $T=1,06 x<3 \mathrm{rad} \mathrm{s}^{-1}$. We observe that the charts vary with the average approximation. For the analysis of the possible approximations in the frequency domain, it is necessary to formulate one or more optimization criteria. In this perspective, two indices are studied:
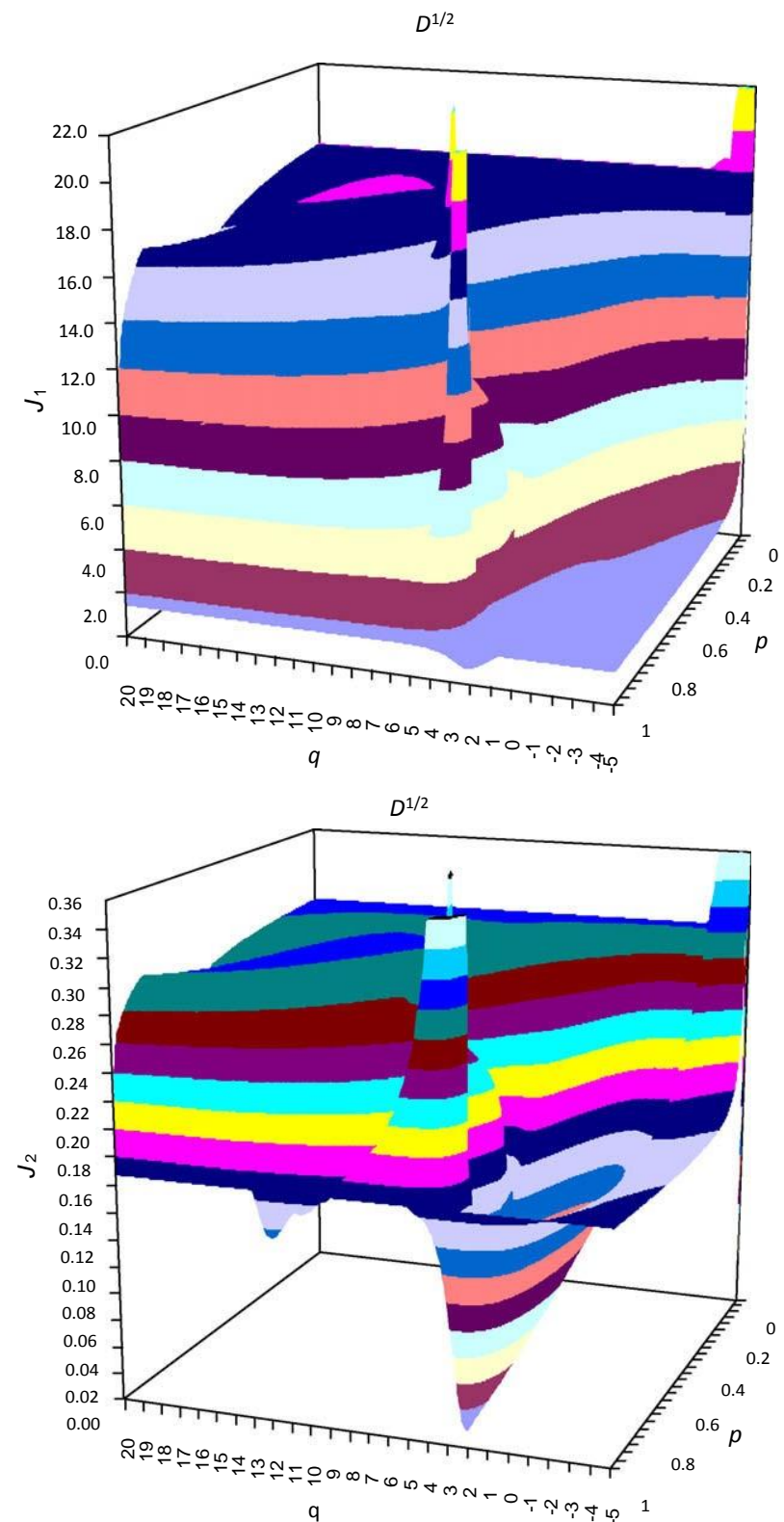

Fig. 3. Locus of $J_{i}(q, p), i=1,2$, for $D^{1 / 2}$ approximated through Padé fractions $H_{k}(j X), k=4$, based on the averaging formulae (6), with $T=1,06 X<3$ rad s ${ }^{-1}$, $N_{X}=300$. 

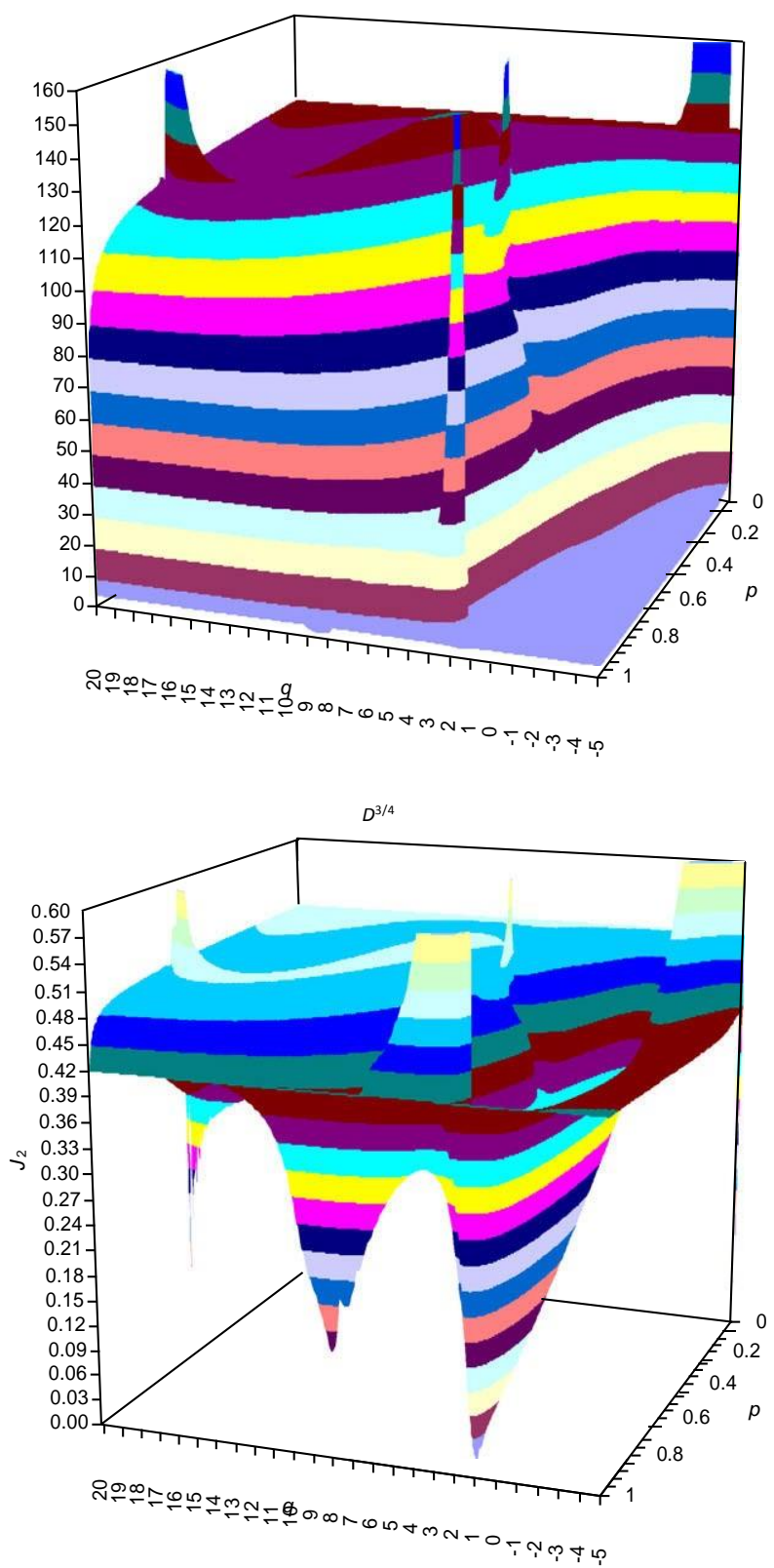

Fig. 4. Locus of $J_{i}(q, p), i=1,2$, for $D^{3 / 4}$ approximated through Padé fractions $H_{k}(j X), k=4$, based on the averaging formulae (6), with $T=1,06 X<3$ rad $\mathrm{s}^{-1}$, $N x=300$.

$$
\begin{aligned}
& J_{1}=\min _{p} \frac{1}{N_{\Omega}} \sum_{i=1}^{n}\left\langle\left\{\operatorname{Re}\left[\left(j \Omega_{i}\right)^{\alpha}\right]-\operatorname{Re}\left[H_{k}\left(\Omega_{i}\right)\right]\right\}^{2}+\left\{\operatorname{Im}\left[\left(j \Omega_{i}\right)^{\alpha}\right]-\operatorname{Im}\left[H_{k}\left(\Omega_{i}\right)\right]\right\}^{2}\right\rangle, \\
& J_{2}=\min _{p} \frac{1}{N_{\Omega}} \sum_{i=1}^{n} \frac{\left\{\operatorname{Re}\left[\left(j \Omega_{i}\right)^{\alpha}\right]-\operatorname{Re}\left[H_{k}\left(j \Omega_{i}\right)\right]\right\}^{2}+\left\{\operatorname{Im}\left[\left(j \Omega_{i}\right)^{\alpha}\right]-\operatorname{Im}\left[H_{k}\left(j \Omega_{i}\right)\right]\right\}^{2}}{\left.\left.\left\{\operatorname{Re}\left[j \Omega_{i}\right)^{\alpha}\right]+\operatorname{Re}\left[H_{k}\left(j \Omega_{i}\right)\right]\right\}^{2}+\left\{\operatorname{Im}\left[j \Omega_{i}\right)^{\alpha}\right]+\operatorname{Im}\left[H_{k}\left(j \Omega_{i}\right)\right]\right\}^{2}}
\end{aligned}
$$


Table 1

Values of the $(q, p)$ domain where occur singular values of $J_{i}(q, p), i=1,2$.

$\begin{array}{llllllll}a & q & p & q & p & q & & \\ & q & 0.918 & 15.1 & 0.546 & & \\ 1 / 4 & 6.2 & 0.012 & 3.1 & 0.930 & 7.35 & 0.496 \\ 3 / 4 & -4.95 & 0.006 & 2.15 & 0.956 & 4.65 & 0.374\end{array}$




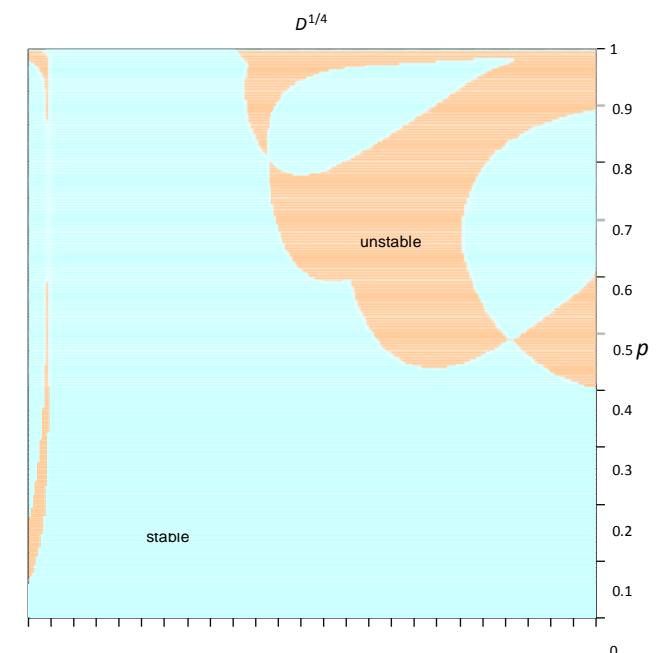

$-5-4-3-2-100112234455678891011121314151617181920$

9

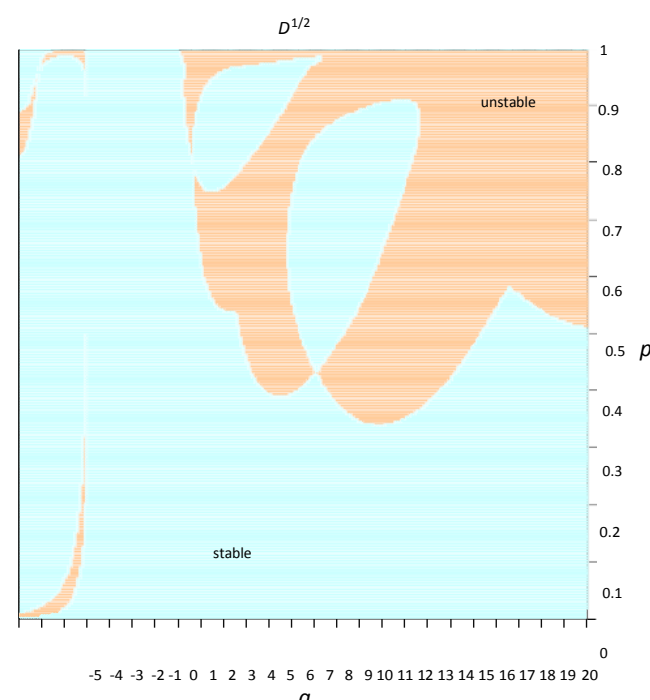

$q$

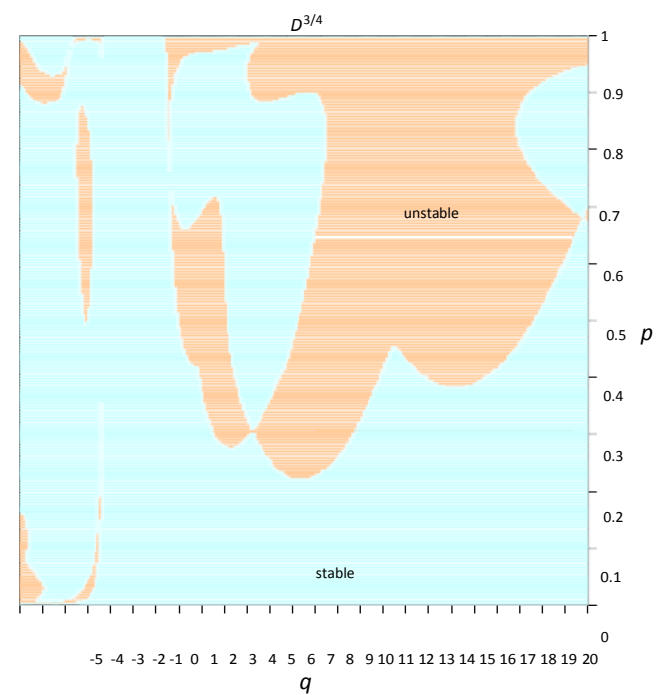

Fig. 5. Locus $S(q, p)$ of stable Padé approximations $H_{k}(j X), k=4$, of $D^{a}, a=\{1 / 4,1 / 2,3 / 4\}$. 
Table 2

Values of $J_{1}$ and $J_{2}$ for $(q, p)=(1,3 / 4)$ and the optimal tuning.

\begin{tabular}{|c|c|c|c|c|c|c|c|c|}
\hline$a$ & $q$ & $p$ & $J_{1}$ & $J_{2}$ & $q$ & $p$ & $J_{1}$ & $J_{2}$ \\
\hline $1 / 4$ & 1 & $3 / 4$ & 0.0346 & 0.00948 & 5.59 & 0.71 & 0.0196 & 0.0067 \\
\hline $1 / 2$ & 1 & $3 / 4$ & 0.1693 & 0.0245 & 2.09 & 0.719 & 0.1194 & 0.0177 \\
\hline $3 / 4$ & 1 & $3 / 4$ & 0.4752 & 0.0435 & 1.598 & 0.794 & 0.4069 & 0.0407 \\
\hline
\end{tabular}
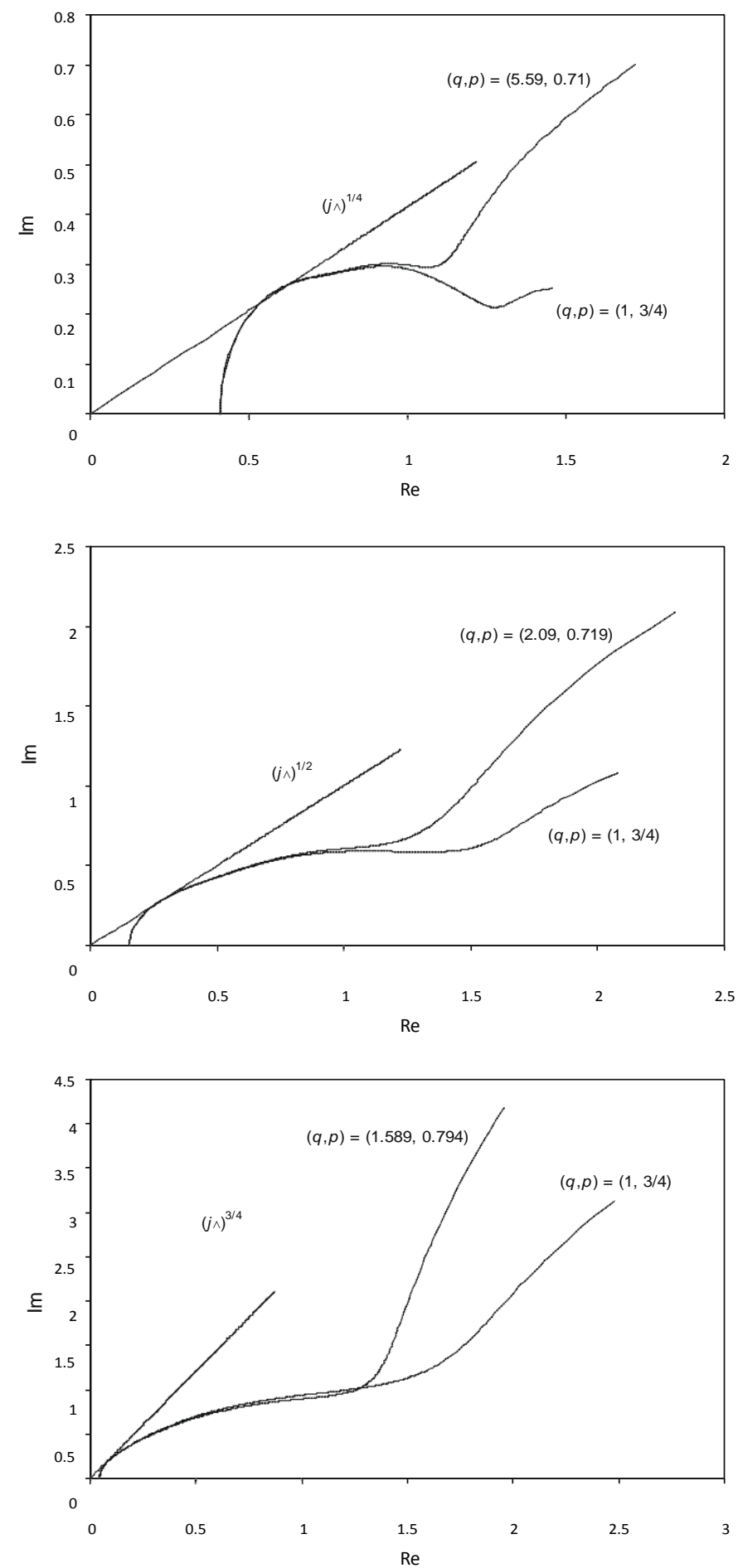

Fig. 6. Polar diagrams of $D^{a}, a=\{1 / 4,1 / 2,3 / 4\}$ : The ideal case $(j X)^{a}$ versus the Padé fractions $H_{k}(j X)$, $k=4$, based on the averaging formulae $(6)$, for $(q, p)=(1$, $3 / 4)$, and the optimal point $(q, p)=(5.59,0.71),(q, p)=(2.09,0.719),(q, p)=(1.589,0.794), T=1,06 X<3 \mathrm{rad} \mathrm{s}^{-1}$. 
where $\left(j X_{i}\right)^{a}$ and $H_{k}\left(j X_{i}\right)$ denote the desired and the $k=4$ order Padé fraction at frequency $X_{i}$, respectively, and Re[ ] and Im[ ] represent the real and imaginary parts, evaluated at the discrete sampling points $X=\left[X_{i}\right], i=0, \ldots, n$. The variable $N_{X}$ rep-resents the number of sampling points and $1 / N_{X}$ is simply a scale factor. In what concerns the optimization functions, $J_{1}$ points to the minimization of the absolute error, while $J_{2}$ is inspired in the minimization of the relative error.

Bearing these ideas in mind, Figs. 2-4 represent the locus of $J_{i}, i=1,2$, for $D^{a}, a=\{1 / 4,1 / 2,3 / 4\}$, versus $(q, p), 06 p 61$, $-56 q 620$, when adopting Padé fractions $H_{k}(j X)$, of $k=4$, based on the averaging formulae (6), for $T=1,06 X<3 \mathrm{rad} \mathrm{s}{ }^{-1}$, $N_{X}=300$.

We verify that the locus of $J_{1}$ and $J_{2}$ have similarities in what concerns the "good" and "bad" regions, and that the cases $a=\{1 / 4$, $1 / 2,3 / 4\}$ lead to charts of the same type. The charts reveal a valley (i.e., the optimal values), roughly with a triangularshape, for the corner region of the $(q, p)$ domain with lower values of $q$, in opposition with the rest of the $(q, p)$ domain wherewe get large values of $J_{i}$, $i=1$, 2. This second non-optimal region is, in fact, composed of two sub-regions separated by a nar- row S-shaped rift. Furthermore, we have also some singular points where the optimization indices reach very high values (i.e., very poor performance). The locus of $J_{1}$ and $J_{2}$ have singular points at the same location (listed in Table 1 ) and, in those cases, the frequency response has a clear divergence.

Besides the optimization of $J_{i}(q, p), i=1,2$, and the avoidance of singular points, we need also to analyze the stability of the resulting $z$-transfer functions. Therefore, Fig. 5 shows the locus $S(q, p)$ of the stable approximations, that is, those having allpoles of the fraction inside the unit circle (i.e., $\left.j z_{i} j<1, i=1, \ldots, 4\right)$.

We verify that the standard parameters $(q, p)=(1,3 / 4)$ are a good general solution. However, we conclude also that we can get better solutions, each one customized for the particular value of $a$. For obtaining the best stable tuning of $(q, p)$, we haveto deplete the locus $J_{i}(q, p), i=1,2$, with the unstable points detected in $S(q, p)$. In this perspective, Table 2 depicts the values of $J_{1}$ and $J_{2}$ for $(q, p)=$ $(1,3 / 4)$ and the optimal parameters when $a=\{1 / 4,1 / 2,3 / 4\}$, while Fig. 6 shows the corresponding fre-quency response. We conclude that we get better results particularly at high frequencies.

The generalized average approach is not restricted to the calculation of fractional derivatives or integrals. In fact, the algo- rithm can be easily used in the fraction approximation of any fractional expression as, for example, the fractional PID con-troller. Therefore, the approximation of any fractional order expression can be tackled through the generalized mean leadingto optimal results.

\section{Conclusions}

The recent advances in fractional calculus point towards important developments in the application of this mathematical concept. During the last years several algorithms for the approximate calculation of fractional derivatives and integrals wereproposed, namely based on Padé fraction expansions. Nevertheless, the resulting expressions are sub-optimal, revealing thatthe approximation should be formulated as an optimization problem. In this paper a new method, based on the generalized average for the calculation of fractional expressions, was proposed. Furthermore, two optimization indices were introduced and several cases were evaluated. The results demonstrate the superior performance of the generalized algorithm.

\section{References}

[1] Oldham Keith B, Spanier J. The fractional calculus: theory and application of differentiation and integration to arbitrary order. New York: Academic Press; 1974.

[2] Ross B. Fractional calculus. Math Mag 1977;50:15-122.

[3] Le Méhauté A. Fractal geometries: theory and applications. London, England: Penton Press; 1991. ISBN:1 857180011.

[4] Samko SG, Kilbas AA, Marichev OI. Fractional integrals and derivatives: theory and applications. New York: Gordon \& Breach; 1993. [5] Miller KS,

Ross B. An introduction to the fractional calculus and fractional differential equations. New York: Wiley; 1993.

[6] Bagley RL, Torvik PJ. Fractional calculus - a different approach to the analysis of viscoelastically damped structures. AIAA J 1983;21:741-8. [7] Oustaloup

A. La commande CRONE: commande robuste d'ordre non entier. Hermes; 1991.

[8] Mainardi Francesco. Fractional relaxation-oscillation and fractional diffusion-wave phenomena. Chaos, Solitons Fractals 1996;7:1461-77. [9] Tenreiro

Machado J. Analysis and design of fractional-order digital control systems. J Syst Anal Model Simulat 1997;27:107-22.

[10] Podlubny I. Fractional differential equations. San Diego: Academic Press; 1999.

[11] Podlubny I. Fractional-order systems and $\mathrm{PI}^{\mathrm{k}} \mathrm{D}^{\prime}$-controllers. IEEE Trans Autom Contr 1999;44(1):208-13. [12]

Tenreiro Machado J. Discrete-time fractional-order controllers. J Fract Calc Appl Anal 2001;4:47-66.

[13] Chen YQ, Moore KL. Discretization schemes for fractional-order differentiators and integrators. IEEE Trans Circ Syst - I: Fundam Theory Appl 2002;49(3):363-7.

[14] Tseng C-C. Design of fractional order digital fir differentiators. IEEE Signal Process Lett 2001:8(3):77-9.

[15] Vinagre BM, Chen YQ, Petras I. Two direct Tustin discretization methods for fractional-order differentiator/integrator. J Franklin Inst $2003 ; 340(5): 349-62$.

[16] Chen YQ, Vinagre BM. A new IIR-type digital fractional order differentiator. Signal Processing 2003;83(11):2359-65.

[17] Barbosa RS, Tenreiro Machado J, Silva MS. Time domain design of fractional differintegrators using least squares approximations. Signal Processing 2006;86(10):2567-81.

[18] Al-Alaoui MA. Novel digital integrator and differentiator. Electron Lett 1993;29(4):376-8.

[19] Al-Alaoui MA. Filling the gap between the bilinear and the backward-difference transforms: an interactive design approach. Int J Electrical Eng Educ 1997;34(4):331-

[20] Smith Jon Michael. Mathematical modeling and digital simulation for engineers and scientists. 2nd ed. New York: Wiley; 1987.

[21] Tenreiro Machado J, Galhano AM. Approximating fractional derivatives in the perspective of system control. In: Nonlinear dynamics. Berlin: Springer, accepted for publication. doi:10.1007/s11071-008-9409-4.

[22] Bibby J. Axiomatisations of the average and a further generalisation of monotonic sequences. Glasgow Math J 1974;15:63-5.

[23] Abramowitz M, Stegun IA, editors. Handbook of mathematical functions with formulas, graphs, and mathematical tables. New York: Dover; 1972. [24] Weisstein EW. CRC concise encyclopedia of mathematics. Boca Raton (FL): CRC Press; 1998. 